\title{
Partial Volume of Copolymers in Mixed Solvents and Volume Contraction in Bulk-Copolymerization
}

\author{
Takeshi FuKudA, Yung-Dae MA, \\ Minoru NAGATA, ${ }^{*}$ and H. INAGAKI \\ Institute for Chemical Research, Kyoto University, \\ Uji, Kyoto 611, Japan \\ *Junior Women's College of Kyoto Prefectural University, \\ Shimogamo, Kyoto 606, Japan
}

(Received May 17, 1982)

\begin{abstract}
On the basis of a simple and unified phenomenological method for treating the excess volume of mixing in multicomponent systems, formulas were developed for the partial molar volume of a copolymer (or a mixture of two polymers) in a mixture of two solvents and for the volume contraction factor in the bulk-copolymerization of two monomers. The possible dependence of the "net" volume of a copolymer on its primary structure was taken into account. The proposed formulas were partly checked against some experimental data, and found satisfactory. The previously proposed formulas for the copolymerization contraction factor seem to be less generally applicable.
\end{abstract}

KEY WORDS Copolymers in Mixed Solvents / Partial Molar Volume / Copolymerization Contraction Factor / Primary Structure Dependence / Concentration Dependence /

Polymerization is usually accompanied by a negative volume change which is important for determining polymerization rates. The contraction factor $\Gamma$, expressed in units of $\mathrm{mL} \mathrm{mol}^{-1}$, is defined by

$$
\Gamma=-\left(\partial U / \partial N_{\mathbf{p}}\right)
$$

where $U$ is the volume of the system, and $N_{\mathrm{P}}$ is the number of moles of monomeric units converted to polymer molecules. The differentiation in eq 1 should be carried out by taking account of the conservation of mass. Given $\Gamma$, one can readily convert the rate of change in volume or density to the rate of polymerization.

There is considerable complexity in regard to the $\Gamma$ factor for copolymerization, but this matter has never been adequately discussed. In the past, it was customarily assumed that $\Gamma$ for a 1-2 binary copolymerization is given by a linear combination of the contraction factors $\Gamma_{1}$ and $\Gamma_{2}$ for the relevant homopolymerizations ${ }^{1-3}$ :

$$
\Gamma=F_{1} \Gamma_{1}+F_{2} \Gamma_{2}
$$

Here $F_{1}\left(=1-F_{2}\right)$ is the mole fraction of monomeric unit 1 in the copolymer. The inadequacy of eq 2 soon became apparent, ${ }^{4,5}$ and Wittmer ${ }^{5,6}$ proposed the equation

$$
\Gamma=F_{11} \Gamma_{1}+F_{22} \Gamma_{2}+F_{12} \Gamma_{12}
$$

or equivalently

$$
\begin{gathered}
\Gamma=F_{1} \Gamma_{1}+F_{2} \Gamma_{2}+F_{12} \Delta \Gamma_{12} \\
\Delta \Gamma_{12}=\Gamma_{12}-\left(\Gamma_{1}+\Gamma_{2}\right) / 2
\end{gathered}
$$

where $F_{i j}$ is the population of $i-j$ chemical bonds in the copolymer, $F_{i i}+\left(F_{12} / 2\right)=F_{i}(i=1$ or 2$)$, and $\Gamma_{12}$ and hence $\Delta \Gamma_{12}$ are constants independent of $F_{i j}$. The basic idea underlying eq 3 is equivalent to that of Inagaki et al., ${ }^{7,8}$ who first noticed that the apparent molar volume of a copolymer in a single solvent differs from the composition average of those of the homopolymers by an amount proportional to $F_{12}$. More recently, Braun et al. ${ }^{9,10}$ and Disselhoff ${ }^{11}$ employed an equation analogous to eq 


\section{T. FuKuda et al.}

3 , but its physical meaning is not very clear.

In this paper, we present unified and theoretically more complete formulas for the partial molar volume of a binary copolymer in a binary mixture of solvents and for the $\Gamma$ factor for the bulkcopolymerization of two monomers. These formulas are derived on the basis of the van LaarScatchard type equation of mixing, which takes into consideration all contributing factors toward the excess volume of mixing, and which provides reasonably adequate explanation of desired quantities as functions of concentration. The possible dependence of volume parameters on the primary structure of copolymers is also taken into account by introducing the diad approximation proposed by Inagaki ${ }^{7,8}$ and Wittmer. ${ }^{5,6}$

Prediction of the $\Gamma$ factor for a given copolymerization on the basis of this rather comprehensive treatment requires an independent determination of a certain number of parameters. If this number is too large, the use of the dilatometric method has little advantage. As for incipient copolymerizations, this number is only two, provided that $\Gamma_{1}, \Gamma_{2}$ and the volumes of the monomer mixtures are known. In contrast, Wittmer's eq $3 \mathrm{~b}$ includes the single parameter $\Delta \Gamma_{12}$. It is shown below that eq $3 \mathrm{~b}$ is correct only in limitted cases. Our formulas are compared with some published data to check validity.

\section{THEORETICAL}

\section{Systems of Homopolymers and Solvents}

We consider a system consisting of $z$ components $(z \geqq 2)$, each of which may be either a homopolymer or a solvent. The volume $U$ of the system is assumed to be given by the van Laar-Scatchard form ${ }^{12}$

$$
\begin{gathered}
U=U^{*}\left(1+\sum_{m<n}^{z} \sum_{m n}^{z} a_{m} \phi_{m} \phi_{n}\right) \\
U^{*}=\sum_{m}^{z} N_{m} V_{m}
\end{gathered}
$$

where $N_{m}$ is the number of moles of component $m$ having a molar volume $V_{m}$ in the pure state (when the component is a polymer, $N_{m}$ is the number of moles of monomeric units, and $V_{m}$ is the molar volume per monomeric unit), $\phi_{m}$ is defined as a volume fraction before mixing, i.e., $\phi_{m}=N_{m} V_{m} / U^{*}$, and $a_{m n}$ is the binary interaction parameter between the components $m$ and $n$.

Recently, Aminabhavi and Munk ${ }^{13}$ successfully treated the excess volume and polarizability of mixing of one polymer and two solvents, using a refined version of eq 4 . Their equation includes a ternary interaction term as well as binary terms, but according to their own experimental results, the contribution from the ternary term was of minor importance. ${ }^{13}$ For simplicity, ternary interactions are not considered here. Generally, the interaction parameter $a_{m n}$ is a function of the relative composition, $\phi_{m} /\left(\phi_{m}+\phi_{n}\right)$. As a crude approximation, we assume throughout this paper that all $a_{m n}$ are constants, although it is not very difficult to incorporate the composition dependence of $a_{m n}$ into the theory.

The partial molar volume $\tilde{V}_{i}$ of component $i$ is obtained by differentiating $U$ with respect to $N_{i}$ with all $N_{j}(j \neq i)$ fixed constant:

$$
\begin{aligned}
\widetilde{V}_{i} & =\left(\partial U / \partial N_{i}\right)_{N_{j}} \\
& =V_{i}\left(1+\sum_{m \neq i}^{z} a_{i m} \phi_{m}-\sum_{m<n}^{z} \sum_{m n}^{z} a_{m n} \phi_{m} \phi_{n}\right)
\end{aligned}
$$

\section{Systems of One Copolymer and Two Solvents}

Next we consider a system consisting of a 1-2 binary copolymer and two solvents (components 3 and 4). We assume that the interactions in this system are the same as in the system made up by mixing homopolymers 1 and 2 with solvents 3 and 4 in such a way that the volume fractions are the same as in the copolymer system. Thus we may write $U$ as

$$
U=U^{*}\left(1+\sum_{n<m}^{4} \sum_{m n}^{4} a_{m n} \phi_{m} \phi_{n}\right)
$$

The reference volume $U^{*}$ may be properly written

$$
\begin{gathered}
U^{*}=N_{\mathrm{P}} V_{\mathrm{P}}{ }^{*}+N_{\mathrm{S}} V_{\mathrm{S}}^{*} \\
N_{\mathrm{P}}=N_{1}+N_{2} \\
N_{\mathrm{S}}=N_{3}+N_{4}
\end{gathered}
$$

where $V_{\mathrm{S}}{ }^{*}$ is a reference molar volume of the solvent mixture,

$$
\begin{aligned}
& V_{\mathrm{S}}{ }^{*}=f_{3} V_{3}+f_{4} V_{4} \\
& f_{3}=1-f_{4}=N_{3} / N_{\mathrm{S}}
\end{aligned}
$$

and $V_{\mathrm{P}} *$ is a reference molar volume (per monomeric unit) of the copolymer, which, as indicated 
previously, is assumed to be given by

$$
\begin{gathered}
V_{\mathrm{P}}{ }^{*}=F_{1} V_{1}+F_{2} V_{2}+F_{12} \Delta V_{12} \\
F_{1}=1-F_{2}=N_{1} / N_{\mathrm{P}}
\end{gathered}
$$

where $\Delta V_{12}$ is a constant, and $F_{12}$ is the relative population of 1-2 chemical bonds in the copolymer. All the equations for $\Delta V_{12}=0$ are identical with those for $F_{12}=0$, or the equations for the twopolymers-two-solvents system formulated in a previous section. In reality, copolymer systems, even if $\Delta V_{12}=0$, may be different from homopolymer systems. For example, phase-separation may occur in one system but not necessarily in another, and this brings about a certain difference in the two systems. Obviously, this contradiction arises from the basic assumption involved in eq 4 and 7 that all components mix with each other randomly. Care should be taken in this matter, when data for one system are compared with those for another.

Now, we introduce the volume fractions $\phi_{\mathrm{P}}$ and $\phi_{\mathrm{S}}$ of the copolymer and solvents, respectively, which are represented by

$$
\begin{aligned}
& \phi_{\mathrm{P}}=\phi_{1}+\phi_{2}=N_{\mathrm{P}} V_{\mathrm{P}}{ }^{*} / U^{*} \\
& \phi_{\mathrm{S}}=\phi_{3}+\phi_{4}=N_{\mathrm{S}} V_{\mathrm{S}}{ }^{*} / U^{*}
\end{aligned}
$$

Clearly, $\phi_{\mathrm{P}}+\phi_{\mathrm{S}}=1$. We further introduce the volume-based compositions $X_{i}(i=1$ or 2$)$ and $x_{j}$ $(j=3$ or 4$)$ of the copolymer and the solvent mixture, respectively, which are represented by

$$
\begin{aligned}
X_{1} & =1-X_{2}=\phi_{1} / \phi_{\mathrm{P}} \\
x_{3} & =1-x_{4}=\phi_{3} / \phi_{\mathrm{S}} \\
& =f_{3} V_{3} / V_{\mathrm{S}}{ }^{*}
\end{aligned}
$$

The copolymer volume composition $X_{1}$ may be legitimately given by

$$
\left.X_{1}=\left[F_{1} V_{1}+F_{12} \Delta V_{12}\left(\frac{V_{1}}{V_{1}+V_{2}}\right)\right)\right] / V_{\mathrm{P}}^{*}
$$

We note that $V_{\mathbf{P}}{ }^{*}$ defined by eq 13 is not the molar volume in the pure state. In the persent treatment ( $c f$. eq 7,8 , and 17), the pure-state volume $V_{\mathbf{P}}$ of the copolymer is given by

$$
V_{\mathrm{P}}=V_{\mathrm{P}} *\left(1+a_{12} X_{1} X_{2}\right)
$$

Similarly, the molar volume of the solvent mixture (in the absence of a polymer) is given by

$$
V_{\mathrm{S}}=V_{\mathrm{s}}^{*}\left(1+a_{34} x_{3} x_{4}\right)
$$

The partial molar volume $\tilde{V}_{\mathrm{P}}$ of the copolymer (per monomeric unit) is given as

$$
\begin{gathered}
\tilde{V}_{\mathrm{P}}=\left(\partial U / \partial N_{\mathrm{P}}\right)_{X_{1}, N_{3}, N_{4}} \\
=V_{\mathrm{P}}{ }^{*}\left[1+A(X, x) \phi_{\mathrm{S}}{ }^{2}-a_{34} x_{3} x_{4} \phi_{\mathrm{S}}{ }^{2}\right. \\
\left.+a_{12} X_{1} X_{2} \phi_{\mathrm{P}}\left(1+\phi_{\mathrm{S}}\right)\right] \\
A(X, x)=a_{13} X_{1} x_{3}+a_{14} X_{1} x_{4} \\
+a_{23} X_{2} x_{3}+a_{24} X_{2} x_{4}
\end{gathered}
$$

Equation 22 can be expressed in terms of $\tilde{V}_{i, j}$, the partial molar volume of $i$ in the $i-j$ two-component system having the same solvent fraction $\phi_{\mathrm{s}}$. Referring to eq 6 , we have $\tilde{V}_{i, j}=V_{i}\left(1+a_{i j} \phi_{j}^{2}\right)$ with $\phi_{j}=$ $1-\phi_{i}=\phi_{\mathrm{S}}$. Thus,

$$
\begin{aligned}
\tilde{V}_{\mathrm{P}}= & x_{3}\left(F_{1} \tilde{V}_{1,3}+F_{2} \tilde{V}_{2,3}\right)+x_{4}\left(F_{1} \tilde{V}_{1,4}+F_{2} \tilde{V}_{2,4}\right) \\
& +F_{12} \Delta V_{12}-V_{\mathrm{P}}{ }^{*}\left[a_{34} x_{3} x_{4} \phi_{\mathrm{S}}{ }^{2}\right. \\
& \left.-a_{12} X_{1} X_{2} \phi_{\mathrm{P}}\left(1+\phi_{\mathrm{S}}\right)\right]+\Delta
\end{aligned}
$$

where the last term $\Delta$ is a linear function of $a_{i j} \Delta V_{12}$, which is generally so small compared with other terms that it may be neglected. In a similar approximation, the $V_{\mathrm{P}} *$ in the fourth term in eq 24 may be equated to $\tilde{V}_{\mathrm{P}}$. We thus have

$\tilde{V}_{\mathrm{P}}=$

$\frac{x_{3}\left(F_{1} \tilde{V}_{1,3}+F_{2} \tilde{V}_{2,3}\right)+x_{4}\left(F_{1} \tilde{V}_{1,4}+F_{2} \tilde{V}_{2,4}\right)+F_{12} \Delta V_{12}}{1+a_{34} x_{3} x_{4} \phi_{\mathrm{S}}^{2}-a_{12} X_{1} X_{2} \phi_{\mathrm{P}}\left(1+\phi_{\mathrm{S}}\right)}$

In the present treatment, we have assumed $\Delta V_{12}$ and $a_{12}$ to be constants independent of the solvents. To determine $\Delta V_{12}$, it may be convenient to measure a copolymer in a single solvent, say solvent 3 , in the dilute limit, i.e., $\phi_{\mathrm{P}} \rightarrow 0$. In this case, eq 25 reduces to

$$
\tilde{V}_{\mathrm{P}, 3}{ }^{\circ}=F_{1} \tilde{V}_{1,3}{ }^{\circ}+F_{2} \tilde{V}_{2,3}{ }^{\circ}+F_{12} \Delta V_{12}
$$

Hereafter, the superscript ' $O$ ' denotes the dilute limit. Equation 26 is equivalent to the expression proposed by Inagaki et al. ${ }^{7,8}$ for the "apparent" molar volume of a copolymer in a solvent. The parameter $a_{12}$ may be determined by the study of either a copolymer or a mixture of the homopolymers $\left(F_{12}=0\right)$ in a single solvent at finite concentrations:

$$
\tilde{V}_{\mathrm{P}, 3}=\frac{F_{1} \tilde{V}_{1,3}+F_{2} \tilde{V}_{2,3}+F_{12} \Delta V_{12}}{1-a_{12} X_{1} X_{2} \phi_{\mathrm{P}}\left(1+\phi_{\mathrm{S}}\right)}
$$




\section{T. FuKUdA et al.}

Such a study has recently been carried out by Nagata et al. ${ }^{14}$ for a two-polymers-one-solvent system. Finally, the parameter $a_{34}$ in eq 25 may be determined by the study of the solvent mixtures ( $c f$. eq 21). The importance of this term has been demonstrated by Aminabhavi and Munk ${ }^{13}$ for onepolymer-two-solvents systems. Equation 25 (more rigorously eq 24) with $F_{2}=F_{12}=\Delta V_{12}=\phi_{\mathrm{P}}=0$ reduces to their equation with the ternary interaction term omitted.

\section{The Contraction Factor for Bulk-Copolymerization}

In this section, we consider a system of a 1-2 binary copolymer and two monomers 1 and 2 . The equations derived above are valid if solvents 3 and 4 are regarded as monomers 1 and 2, respectively.

However, some additional parameters may be necessary. In a copolymerization, monomers are continually converted to polymers, satisfying the following relations at any instant:

$$
N_{j}^{\circ}=N_{i}+N_{j} ; \quad(i, j)=(1,3) \text { or }(2,4)
$$

Here $N_{i}$ is the total number of moles of monomer $j$ converted to polymers, and $N_{j}$ and $N_{j}^{\circ}$ are the numbers of moles of monomer $j$ present at a given instant and at the onset of the polymerization, respectively. Since conversion rates are generally different for the two monomers, the compositions of the copolymer and the feed continually change. We let $\bar{F}_{i}$ be the average mole-composition for the copolymers that have been produced up to the instant in question; and $F_{i}$ be the composition of the copolymer produced at that particular instant. Clearly,

$$
\begin{aligned}
& \bar{F}_{i}=N_{i} / N_{\mathrm{P}} \\
& F_{i}=\mathrm{d} N_{i} / \mathrm{d} N_{\mathrm{P}}
\end{aligned}
$$

with $N_{\mathrm{P}}=N_{1}+N_{2}$, as before. The average and instantaneous values of all other relevant quantities are distinguished by attaching the symbol '-' to the former. Clearly, the volume $U$ of the system is given by eq 7 with $U^{*}$ and all $\phi_{m}$ expressed in terms of the average quantities. For example, the reference volume of the copolymer to be used in eq 8 is $\bar{V}_{\mathrm{P}}{ }^{*}$ given by

$$
\bar{V}_{\mathrm{P}}{ }^{*}=\bar{F}_{1} V_{1}+\bar{F}_{2} V_{2}+\bar{F}_{12} \Delta V_{12}
$$

The contraction factor $\Gamma$, defined as a decrease in the volume of the system per unit mole of the monomers polymerized, is obtained as

$$
\begin{aligned}
\Gamma= & -\left(\partial U / \partial N_{\mathrm{P}}\right)_{N_{3}{ }^{\circ}, N_{4}{ }^{\circ}} \\
= & \left(F_{1} V_{3}+F_{2} V_{4}-V_{\mathrm{P}}{ }^{*}\right)\left[1-a_{34} x_{3} x_{4} \phi_{\mathrm{S}}{ }^{2}\right. \\
& \left.-A(\bar{X}, x) \phi_{\mathrm{S}} \phi_{\mathrm{P}}-a_{12} \bar{X}_{1} \bar{X}_{2} \phi_{\mathrm{P}}{ }^{2}\right] \\
& -\phi_{\mathrm{S}}\left[A(X, x) V_{\mathrm{P}}{ }^{*}-a_{34}\left(x_{4} F_{1} V_{3}+x_{3} F_{2} V_{4}\right)\right] \\
& -\phi_{\mathrm{P}}\left[a_{12} V_{\mathrm{P}}{ }^{*}\left(X_{1} \bar{X}_{2}+X_{2} \bar{X}_{1}\right)\right. \\
& -\bar{X}_{1}\left(a_{13} F_{1} V_{3}+a_{14} F_{2} V_{4}\right) \\
& \left.-\bar{X}_{2}\left(a_{23} F_{1} V_{3}+a_{24} F_{2} V_{4}\right)\right]
\end{aligned}
$$

In the dilute limit of polymer concentration, eq 32 converges to

$$
\begin{aligned}
\Gamma^{\circ}= & \left(F_{1} V_{3}+F_{2} V_{4}-V_{\mathrm{P}}^{*}\right)\left(1-a_{34} x_{3} x_{4}\right) \\
& -A(X, x) V_{\mathrm{P}}{ }^{*}+a_{34}\left(x_{4} F_{1} V_{3}+x_{3} F_{2} V_{4}\right)
\end{aligned}
$$

By neglecting trivial terms like $a_{i j} \Delta V_{12}$ and introducing the contraction factors $\Gamma_{1}{ }^{\circ}$ and $\Gamma_{2}{ }^{\circ}$ for the homopolymerizations, eq 33 can be written

$$
\begin{aligned}
\Gamma^{\circ}= & \left(F_{1} \Gamma_{1}{ }^{\circ}+F_{2} \Gamma_{2}{ }^{\circ}\right)\left(1-a_{34} x_{3} x_{4}\right)-F_{12} \Delta V_{12} \\
& +x_{4} F_{1}\left(a_{34} V_{3}-\Delta a_{1} V_{1}\right) \\
& +x_{3} F_{2}\left(a_{34} V_{4}-\Delta a_{2} V_{2}\right) \\
& \Delta a_{1}=a_{14}-a_{13} ; \quad \Delta a_{2}=a_{23}-a_{24}
\end{aligned}
$$

where use of the following relations have been made, from which $\Gamma_{i}{ }^{\circ}$ is obtained by setting $\phi_{i}=$ $1-\phi_{j}=0$ :

$$
\begin{gathered}
\Gamma_{i}=V_{j}-V_{i}+a_{i j}\left(\phi_{i}{ }^{2} V_{j}-\phi_{j}{ }^{2} V_{i}\right) \\
(i, j)=(1,3) \text { or }(2,4) ; \phi_{i}+\phi_{j}=1
\end{gathered}
$$

Equation 34 can be converted to a more useful form. If the terminal-model kinetics of copolymerization $^{15-17}$ is valid, ${ }^{18}$ the well-known relations between the copolymer structure and feed composition hold, e.g.

$$
\begin{gathered}
F_{1}=\frac{f_{3}\left(r_{1} f_{3}+f_{4}\right)}{f_{3}\left(r_{1} f_{3}+f_{4}\right)+f_{4}\left(r_{2} f_{4}+f_{3}\right)} \\
F_{12}=\frac{2 f_{3} f_{4}}{f_{3}\left(r_{1} f_{3}+f_{4}\right)+f_{4}\left(r_{2} f_{4}+f_{3}\right)}
\end{gathered}
$$

where $r_{1}$ and $r_{2}$ are the reactivity ratios (in the usual notation, $f_{3}$ and $f_{4}$ are written as $f_{1}$ and $f_{2}$, respectively, but to avoid confusion, we adopt the present notation). Use of eq 37 and 38 in eq 34 gives

$\Gamma^{\circ}=\left(F_{1} \Gamma_{1}^{\circ}+F_{2} \Gamma_{2}^{\circ}\right)\left(1-a_{34} x_{3} x_{4}\right)+G(f) R(f)$ 


$$
\begin{gathered}
G(f)=F_{12} /\left[f_{3}+f_{4}\left(V_{4} / V_{3}\right)\right] \\
R(f)=I+S f_{3}
\end{gathered}
$$

where $I$ and $S$ are constants independent of $f_{3}$ :

$$
\begin{aligned}
I= & -\left(V_{4} / V_{3}\right) \Delta V_{12} \\
& +\left(V_{4} / 2 V_{3}\right)\left(a_{34} V_{3}-\Delta a_{1} V_{1}\right) \\
& +\left(r_{2} / 2\right)\left(a_{34} V_{4}-\Delta a_{2} V_{2}\right) \\
S= & -\left[1-\left(V_{4} / V_{3}\right)\right] \Delta V_{12} \\
& +\left(r_{1}-1\right)\left(V_{4} / 2 V_{3}\right)\left(a_{34} V_{3}-\Delta a_{1} V_{1}\right) \\
& -\left[\left(r_{2}-1\right) / 2\right]\left(a_{34} V_{4}-\Delta a_{2} V_{2}\right)
\end{aligned}
$$

Thus, the function $R$ given by

$$
R(f)=\left[\Gamma^{\circ}-\left(F_{1} \Gamma_{1}{ }^{\circ}+F_{2} \Gamma_{2}{ }^{\circ}\right)\left(1-a_{34} x_{3} x_{4}\right)\right] / G(f)
$$

is linear in $f_{3}$ (cf. eq 41). Provided that $\Gamma_{1}^{\circ}, \Gamma_{2}^{\circ}, a_{34}$ and the elementary parameters in eq 40 are known, measurements of $\Gamma^{\circ}$ at two different values of $f_{3}$ suffice to predict $\Gamma^{\circ}$ at any other values of $f_{3}$. For this purpose, there is no need to know the individual parameters in eq 42 and 43. A similar simplification is possible for $\tilde{V}_{\mathrm{P}}{ }^{\circ}$ that can be obtained from eq 25 .

To a crude approximation, we may set $V_{3} \simeq V_{4}$, and neglect the $a_{34}$-term in the first term in eq 39 (but not those in the other terms, whose contributions may be important unless $a_{34}$ is very small). In special cases, e.g., $r_{1}=r_{2}=1$, the equation thus derived becomes formally the same as eq $3 b$.

\section{DISCUSSION}

We have expressed $\tilde{V}_{\mathrm{P}}$ and $\Gamma$ in terms of the parameters $a_{i j}$ and $\Delta V_{12}$. Each of the six $a_{i j}$ parameters is related to the excess volume of physically mixing components $i$ and $j$, whereas $\Delta V_{12}$ is related to the excess volume of "chemically mixing" components 1 and 2, i.e., formation of 1-2 covalent bonds. To our knowledge, $a_{i j}$ as well as $\Delta V_{12} / V_{\mathrm{P}}{ }^{*}$ may be of the order of $10^{-2}$, and their contributions may possibly be equally important. The prediction of $\tilde{V}_{\mathrm{P}}$ or $\Gamma$ based on eq 22 (or 25) or 32 thus requires

Table I. Values of contraction factor and other relevant parameters for low-conversion copolymerizations $\left(60^{\circ} \mathrm{C}\right)^{a}$

\begin{tabular}{cccccc}
\hline ST-MMA: & $r_{1}=0.52$ & $r_{2}=0.46$ & $V_{3}=119.4$ & $V_{4}=111.5$ & $a_{34}=0.00$ \\
\hline$f_{3}$ & 0 & 0.22 & 0.44 & 0.76 & 1 \\
$\Gamma^{\circ}$ & 29.77 & 26.58 & 26.13 & 23.79 & 20.30 \\
\hline BMA-ST: & $r_{1}=0.53$ & $r_{2}=0.34$ & $V_{3}=175.3$ & $V_{4}=119.4$ & $a_{34}=0.004$ \\
\hline$f_{3}$ & 0 & 0.18 & 0.59 & 0.77 & 1 \\
$\Gamma^{\circ}$ & 20.30 & 22.94 & 25.18 & 27.49 & 33.66 \\
\hline BMA-MMA: & $r_{1}=1.06$ & $r_{2}=0.83$ & $V_{3}=175.3$ & $V_{4}=111.5$ & $a_{34}=-0.010$ \\
\hline$f_{3}$ & 0 & 0.11 & 0.39 & 0.76 & 1 \\
$\Gamma^{\circ}$ & 29.77 & 29.71 & 30.49 & 31.88 & 33.66 \\
\hline NVP-ST: & $r_{1}=0.08$ & $r_{2}=24.2$ & $V_{3}=110.2$ & $V_{4}=119.4$ & $a_{34}=-0.041$ \\
\hline$f_{3}$ & 0 & 0.20 & 0.50 & 0.80 & 1 \\
$\Gamma^{\circ}$ & 20.30 & 19.54 & 17.94 & 15.93 & 13.88 \\
\hline NVP-MMA: & $r_{1}=0.15$ & $r_{2}=4.39$ & $V_{3}=110.2$ & $V_{4}=111.5$ & $a_{34}=-0.020$ \\
\hline$f_{3}$ & 0 & 0.20 & 0.50 & 0.80 & 1 \\
$\Gamma^{\circ}$ & 29.32 & 20.13 & 24.00 & 27.52 & 13.88 \\
\hline
\end{tabular}

a Data from ref 9 and 10: In system A-B, components 1, 2, 3, and 4 are poly-A, poly-B, monomer A and monomer B, respectively; $\Gamma^{\circ}$ and $V$ 's are in $\mathrm{ml} \mathrm{mol}^{-1} ; a_{34}$ is an average value. 


\section{T. FUKUDA et al.}

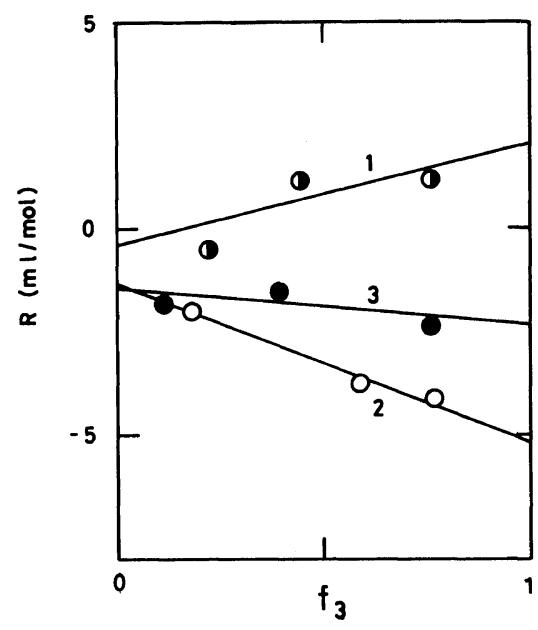

Figure 1. Plot of function $R$ vs. feed composition $f_{3}$ for incipient copolymerization of (1): ST-MMA (half-filled circles), (2): BMA-ST (filled circles), and (3): BMAMMA (open circles). The data were taken from Table I. The solid lines are best-fit representations according to eq 41 .

six $a_{i j}$ and one $\Delta V_{12}$. At present, we are not in a position to test the validity of these general equations, because the experimental data for these seven parameters are not available.

Some discussion can be made on the dilute limit eq 39. Table I shows the data of Braun et al. ${ }^{9,10}$ for low-conversion copolymerizations of (1): styrene (ST) and methyl methacrylate (MMA), (2): butyl methacrylate (BMA) and ST, (3): BMA and MMA, (4): $N$-vinylpyrrolidone (NVP) and ST, and (5): NVP and MMA. The authors determined $\Gamma^{\circ}$ by a direct measurement of the volume vs. conversion curves, but reported it in units different from $\mathrm{mL} \mathrm{mol}^{-1}$. To express their $\Gamma^{\circ}$ in units of $\mathrm{mL} \mathrm{mol}^{-1}$, we employed the parameter values provided by the same authors, and included them in Table I. Equation 39 can now be tested using the experimental data.

In Figure 1, the function $R$ given by eq 44 is plotted against the feed composition $f_{3}$ for systems $1-3$. The data points seem to fall on straight lines, as eq 41 predicts. In system 2, the slope of the line is fairly large, while small in system 3 . According to Wittmer's eq $3 \mathrm{~b}$, the slope in an $R v s . f_{3}$ plot should be nearly zero. According to our equation, this occurs when $r_{1}=r_{2}=1$, as has been pointed out. System 3, in fact, meets this condition approxi- mately. However, these results do not immediately mean a superiority of one equation over the other: First, rather small experimental errors in $\Gamma^{\circ}$ may result in a large slope. Secondly, as far as these systems are concerned, eq $3 \mathrm{~b}$ and 39 can reproduce the data nearly equally as well, if the parameter values are property chosen. In this regard it is evident that eq $3 b$ has an advantage over eq 39 , since the former is a one-parameter theory, and the latter, a two-parameter theory.

More critical tests of these two equations can be made using the data for the remaining two systems. System 4 is particularly interesting, since the reactivity ratios for this system are $r_{1}=0.08$ and $r_{2}=$ 24.2 , and the absolute value of the monomermonomer interaction parameter $a_{34}$ is fairly large $\left(a_{34}=-0.041\right.$ on average). According to eq 42 and 43 , the function $R$ for this system is dominated by the terms involving $r_{2}$ multiplied by $a_{34}$. We thus expect that the intercept, $-I$, as well as the slope, $S$, should be anomalously large. According to eq $3 \mathrm{~b}$, on the other hand, $R$ should be nearly independent of $f_{3}$, and its magnitude should be "normal", i.e., as small as in the previous examples. Figure 2 shows the data for this system. The large scale of the ordinate should be noted. The broken line in the figure has been calculated from eq 42 and 43 using the above values of $r_{i}$ and $a_{34}$ but neglecting all the terms that include the other parameters. It remarkably well reproduces the data points. System 5 is also characterized by relatively large values of $r_{2}$ (=4.39) and $a_{34}(=-0.020$ on average). In this example, nearly one half of the observed dependence of $R$ on $f_{3}$ can be explained by the terms involving $r_{2}$ and $a_{34}$ alone (the dot-dash line in Figure 2). Our equations are thus supported by these data.

Figure 3 illustrates the dependence of $\Gamma^{\circ}$ on copolymer composition $F_{1}$ predicted by various approximate theories. The three curves have been calculated according to eq 2 (broken line), eq $3 \mathrm{~b}$ (dot-dash line) and eq 39 (full line) so as to give the best fit to the data points for system 4 (circles). It is noted that there are very large differences among the $\Gamma^{\circ}$ values predicted by different theories.

Now that the validity of our treatment seems to have been confirmed in part, we proceed to illustrate some behavior of a high-conversion system, for which the dilatometric method may show its greatest power. We take a ST-MMA system as a 


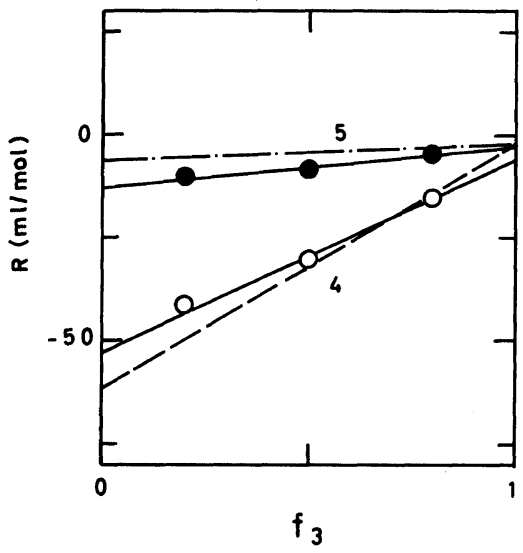

Figure 2. Plot of function $R v s$. feed composition $f_{3}$ for incipient copolymerization of (4): NVP-ST (open circles) and (5): NVP-MMA (filled circles). The data were taken from Table I. The solid lines are best-fit representations according to eq 41 . The broken and dot-dash lines were calculated from eq $41-43$ with $\Delta V_{12}=\Delta a_{1}=\Delta a_{2}=0$ (see the text).

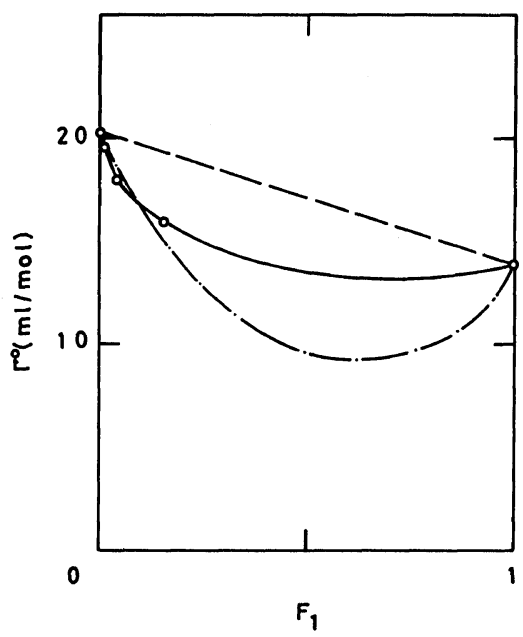

Figure 3. Plot of contraction factor $\Gamma^{\circ} v$ s. copolymer composition $F_{1}$ for incipient copolymerization of NVPST, calculated according to eq 2 (broken line), eq $3 b$ (dot-dash line), and eq 39 (full line) so as to give the best fit to the data points (circles).

model. Values of the basic parameters for this system are listed in Table II. The molar volumes $V_{1}$ and $V_{2}$ of PST (component 1) and PMMA (component 2 ) were tentatively taken from the literature data for the solids. ${ }^{19,20}$ The propriety of this choice will be discussed in a forthcoming paper. ${ }^{14}$ The values of $a_{13}$ and $a_{24}$ were obtained from the above-
Table II. Parameter values employed to calculate the contraction factor for high-conversion copolymerization of ST and MMA $\left(60^{\circ} \mathrm{C}\right)^{\mathrm{a}}$

\begin{tabular}{|c|c|c|c|c|c|c|}
\hline $\begin{array}{c}V_{1}^{\mathrm{b}} \\
100.0\end{array}$ & $\begin{array}{l}V_{2}^{\mathrm{c}} \\
85.4\end{array}$ & $\begin{array}{c}V_{3}{ }^{\mathbf{d}} \\
119.4\end{array}$ & $\begin{array}{c}V_{4}^{\mathrm{d}} \\
111.5\end{array}$ & $\begin{array}{l}\Delta V_{12}{ }^{\mathrm{e}} \\
-1.16\end{array}$ & $\begin{array}{c}r_{1}{ }^{\mathrm{d}} \\
0.52\end{array}$ & $\begin{array}{c}r_{2}{ }^{d} \\
0.46\end{array}$ \\
\hline $\begin{array}{l}a_{12}{ }^{\mathrm{f}} \\
0.00\end{array}$ & $\begin{array}{c}a_{13}{ }^{\mathrm{g}} \\
-0.010\end{array}$ & $\begin{array}{c}a_{14}{ }^{\mathrm{g}} \\
0.040\end{array}$ & $\begin{array}{c}a_{23}{ }^{\mathrm{g}} \\
-0.079\end{array}$ & $\begin{array}{r}a \\
-0\end{array}$ & & $\begin{array}{l}a_{34}{ }^{\mathrm{d}} \\
0.00\end{array}$ \\
\hline
\end{tabular}

a Components 1, 2, 3 and 4 are PST, PMMA, ST, and MMA, respectively; $V$ 's and $\Delta V_{12}$ are in $\mathrm{ml} \mathrm{mol}^{-1}$.

b Reference 19; value for the solid.

c Reference 20; value for the solid.

${ }^{d}$ Reference 9.

e Reference 8; value for $30^{\circ} \mathrm{C}$.

f Reference 14; value for $30^{\circ} \mathrm{C}$.

$\mathrm{g}$ Estimated from the data in ref 9 .

cited data, ${ }^{9}$ which also enabled us to estimate $a_{14}$ and $a_{23}$ (cf. Figure 1 and eq 42 and 43). The value of $a_{12}$ was taken from our results for a PS-PMMAbromobenzene ternary system, ${ }^{14}$ and the $\Delta V_{12^{-}}$ value from the study of Nakazawa et al. for STMMA copolymers in $p$-xylene. ${ }^{8}$ The other parameter values are as given in ref 9 .

Based on eq 32, we are now able to compute $\Gamma$ as a function of the feed-composition $f_{3}$ (at a given instant): all other variables in eq 32 are functions of $f_{3}$ only. For example, the average composition $\bar{F}_{1}$ and the average diad-population $\bar{F}_{12}$ are given by

$$
\begin{gathered}
\bar{F}_{1}=\left(f_{3}^{\circ}-f_{3}\right) / C+f_{3} \\
\bar{F}_{12}=C^{-1} \int_{f_{3}}^{f_{3}} \frac{(1-C) F_{12}}{f_{3}-F_{1}} \mathrm{~d} f_{3}
\end{gathered}
$$

where $f_{3}^{\circ}$ is the composition of the initial feed, and $C$ is the mole-conversion, which is related to $f_{3}$ by $^{21}$

$$
C=1-\left(f_{3} / f_{3}^{\circ}\right)^{\alpha}\left(f_{4} / f_{4}^{\circ}\right)^{\beta}\left[\left(f_{3}^{\circ}-\delta\right) /\left(f_{3}-\delta\right)\right]^{\gamma}
$$

In eq $47, \alpha, \beta, \gamma$, and $\delta$ are known functions of $r_{1}$ and $r_{2}$ only (see ref 21). The integration in eq 46 seems difficult to perform analytically. For the present purpose, it suffices to approximate $\bar{F}_{12}$ by

$$
\bar{F}_{12}=\frac{4 \bar{F}_{1} \bar{F}_{2}}{1+\left[1+4 \bar{F}_{1} \bar{F}_{2}\left(r_{1} r_{2}-1\right)\right]^{1 / 2}}
$$

which is correct for instantaneous copolymers. ${ }^{22} \mathrm{On}$ the other hand, the volume of the system, $U$, is also a function of $f_{3}$ alone. It is thus possible to determine $\Gamma$ as a function of $U$, or a relative change $\Delta U / U^{\circ}$, where $U^{\circ}$ is the initial volume, and $\Delta U=$ 


\section{T. FuKUDA et al.}

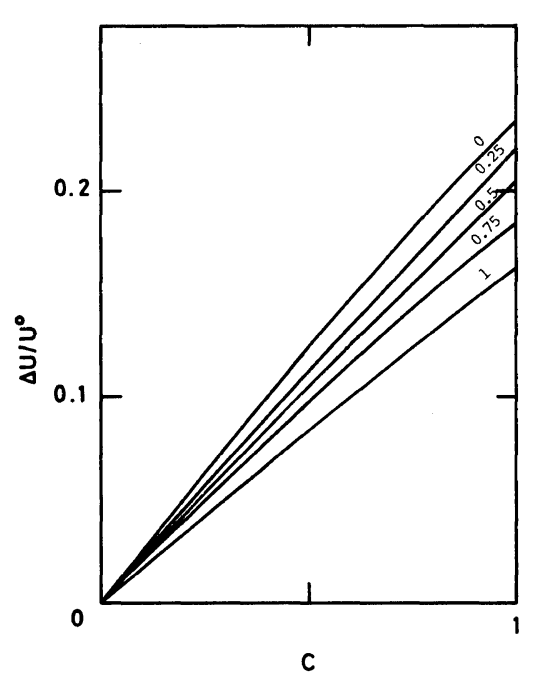

Figure 4. Plot of relative volume change $\Delta U / U^{\circ} v s$. mole conversion $C$ for ST-MMA copolymerization, calculated with the data in Table II for the values of initial ST-composition $f_{3}{ }^{\circ}$ indicated in the figure.

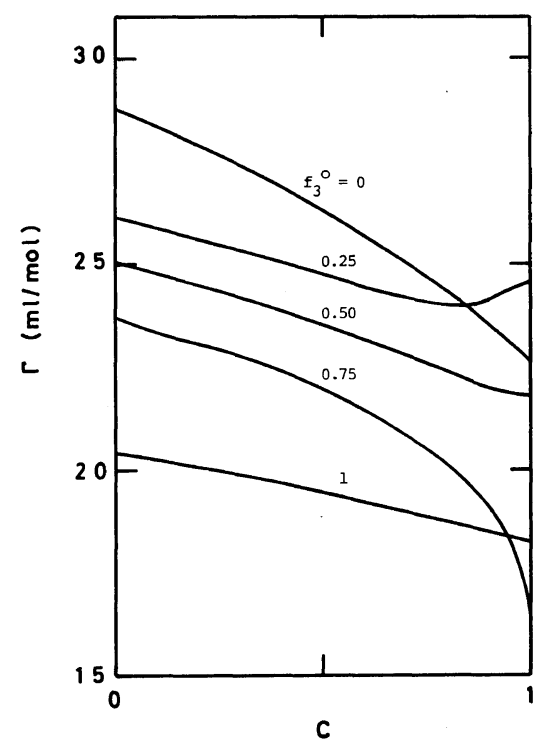

Figure 5. Plot of contraction factor $\Gamma v s$. mole conversion $C$ for ST-MMA copolymerization, calculated with the data in Table II for the values of initial STcomposition $f_{3}^{\circ}$ indicated in the figure.

$U^{\circ}-U$.

The calculated results for several values of initial ST-composition $f_{3}^{\circ}$ are presented in Figures 4 and 5 . Figure 4 gives $\Delta U / U^{\circ}$ and Figure 5 gives $\Gamma$ as a function of $C$. Although not too much importance should be attached to the numerical results, since the parameter values employed may possibly contain errors (in particular, the values of $a_{14}$ and $a_{23}$ seem somewhat unreasonable), these figures serve to illustrate complicated, not easily predictable dependence of $\Gamma$ on $\Delta U / U^{\circ}$ or $C$ as well as on $f_{3}^{\circ}$. No simple approximation such as those expressed by eq 2 and $3 \mathrm{~b}$ seems valid at any level of conversion. A set of accurately estimated values of the parameters for the same system at $40^{\circ} \mathrm{C}$ will be reported elsewhere. ${ }^{23}$

\section{LIST OF SYMBOLS}

\section{General}

$\bar{Y} \quad$ value of $Y$ averaged over different copolymer species

$\tilde{Y} \quad$ partial value of $Y$ in multicomponent solution $Y^{*} \quad$ value of $Y$ under the ideal mixing

$Y^{\circ} \quad$ value of $Y$ at vanishing polymer concentration

\section{Individual}

$A \quad$ a function of $a_{i j}, X_{i}$, and $x_{j}$ (eq 23)

$a_{i j} \quad$ binary interaction parameter related to excess volume of mixing (eq 4)

$\Delta a_{i} \quad$ difference between the binary interaction parameters (eq 35)

$C$ mole conversion

$F_{i}$ mole composition of $i$ in the copolymer: $F_{1}+F_{2}=1$ (eq 14)

$f_{j} \quad$ mole composition of $j$ in the solvent mixture: $f_{3}+f_{4}=1$ (eq 12)

$F_{i j} \quad$ population of $i-j$ chemical bonds in the copolymer: $F_{11}+F_{22}+F_{12}=1$

$N_{i} \quad$ number of moles of component $i$

$N_{\mathrm{P}}$ total number of moles of monomeric units in the copolymer (eq 9)

$N_{\mathrm{S}} \quad$ total number of moles of solvent molecules (eq 10)

$r_{i} \quad$ copolymerization reactivity ratio

$U$ total volume of the system

$V_{i}$ molar volume of component $i$ (in the pure state)

$\tilde{V}_{i, j} \quad$ value of $\tilde{V}_{i}$ in the $i-j$ two component system

$V_{\mathbf{P}}$ molar volume of copolymer per monomeric unit (in the pure state, eq 20)

$V_{\mathrm{P}} *$ reference molar volume of copolymer per monomeric unit (eq 13)

$V_{\mathrm{S}} \quad$ molar volume of the solvent mixture (eq 21) 
$\Delta V_{12}$ correction parameter related to $V_{\mathrm{P}} *$ (eq 13)

$X_{i}$ volume composition of $i$ in the copolymer: $X_{1}+X_{2}=1$ (eq 17)

$x_{j} \quad$ volume composition of $j$ in the solvent mixture: $x_{3}+x_{4}=1$ (eq 18)

$\Gamma$ molar contraction in copolymerization

$\Gamma_{i}$ molar contraction in homopolymerization of monomer $i$

$\Delta \Gamma_{12}$ correction parameter related to $\Gamma$ (eq $3 \mathrm{~b}$ )

$\phi_{i} \quad$ volume fraction of component $i$ in the system

$\phi_{\mathbf{P}} \quad$ volume fraction of copolymer in the system (eq 15)

$\phi_{\mathrm{S}} \quad$ volume fraction of solvents in the system (eq 16)

\section{REFERENCES}

1. H. W. Melville, B. Noble, and W. F. Watson, $J$. Polym. Sci., 2, 229 (1947).

2. S. Loshaek and J. Broderick, J. Polym. Sci., 39, 241 (1959).

3. Z. Izumi, H. Kiuchi, and M. Watanabe, J. Polym. Sci., A, 1, 705 (1963).

4. D. Braun, T. O. Ahn, and W. Kern, Makromol. Chem., 53, 154 (1962).

5. P. Wittmer, Angew. Makromol. Chem., 39, 35 (1974).

6. P. Wittmer, Makromol. Chem., Suppl., 3, 129 (1979).

7. H. Inagaki, Makromol. Chem., 75, 217 (1964).
8. A. Nakazawa, Y. Murakami, T. Kotaka, and H. Inagaki, Bull. Inst. Chem. Res., Kyoto Univ., 53, 387 (1975).

9. D. Braun and G. Disselhoff, Polymer, 18, 963 (1977).

10. D. Braun, G. Disselhoff, and F. Quella, Makromol. Chem., 179, 1239 (1978).

11. G. Disselhoff, Polymer, 19, 111 (1978).

12. G. Scatchard, Trans. Faraday Soc., 33, 160 (1937).

13. T. M. Aminabhavi and P. Munk, Macromolecules, 12, 1186 (1979).

14. M. Nagata, T. Fukuda, and H. Inagaki, to be published.

15. F. R. Mayo and F. M. Lewis, J. Am. Chem. Soc., 66, 1954 (1944).

16. F. T. Wall, J. Am. Chem. Soc., 66, 2050 (1944).

17. T. Alfrey, Jr., and G. Goldfinger, J. Chem. Phys., 12, 205 (1944).

18. T. Fukuda, Y.-D. Ma, and H. Inagaki, Polym. J., 14, 705 (1982).

19. W. Patnode and W. J. Scheiber, J. Am. Chem. Soc., 61, 3449 (1939).

20. J. Brandrup and E. H. Immergut, Eds., "Polymer Handbook," Willey, New York, 1975.

21. V. E. Mayer and G. G. Lowry, J. Polym. Sci., A, 3, 2843 (1965).

22. H. J. Harwood and W. M. Ritchey, J. Polym. Sci., $B, 2,601$ (1964).

23. Y.-D. Ma, T. Fukuda, and H. Inagaki, to be published. 